\title{
Continuous progression of hemorrhage of sphenoid ridge meningioma causing cerebral hernia: A case report and literature review
}

\author{
SONG HAN ${ }^{1}$, YAKUN YANG ${ }^{1}$, ZUOCHENG YANG $^{1}$, NING LIU $^{1}$, \\ $\mathrm{XUELING}^{2}{ }^{2}, \mathrm{CHANGXIANG} \mathrm{YAN}^{1}$ and CHUNJIANG YU ${ }^{1}$ \\ Departments of ${ }^{1}$ Neurosurgery and ${ }^{2}$ Pathology, Sanbo Brain Hospital, \\ Capital Medical University, Beijing 100093, P.R. China
}

Received October 18, 2019; Accepted March 5, 2020

DOI: $10.3892 / \mathrm{ol} .2020 .11590$

\begin{abstract}
The aim of the present study was to explore the clinical characteristics of repeated hemorrhages of meningioma and analyze the causes of hemorrhage. Meningiomas are mostly benign tumors that rarely manifest hemorrhagic strokes. In the present study, a case of sphenoid ridge meningioma with repeated hemorrhages is reported. Internal hemorrhage was first observed, which, on further aggravation, formed a hematoma in the brain parenchyma and finally led to the development of a hernia. No neurological deficit was present after surgery and rehabilitation. A postoperative pathological examination showed increased levels of Ki-67, abnormal blood vessels in the tumors and the presence of progesterone, which indicate possible causes of the hemorrhage. A review of associated previous studies revealed that hemorrhages originate mainly from inside the meningioma. Two cases of meningiomas with repeated hemorrhages have been reported; one in the foramen magnum region and the other in the pineal gland area. The foramen magnum tumor had an interval of 1.33 months between two hemorrhagic episodes. Collecting relevant data from the latter case was not possible. In the present case report, the interval between two bleeding episodes was 3 days. The literature review also revealed that the average age of onset of meningioma is relatively young at only $28.00 \pm 6.24$ years. In conclusion, repeated hemorrhages in meningiomas are extremely rare and the causes have not yet been identified. Increased Ki-67 and abnormally proliferating blood vessels may be potential causes of hemorrhage. Early diagnosis and rapid surgical
\end{abstract}

Correspondence to: $\mathrm{Dr}$ Chunjiang Yu, Department of Neurosurgery, Sanbo Brain Hospital, Capital Medical University, 50 Xiang Shan Yi Ke Song Road, Haidian, Beijing 100093, P.R. China

E-mail: yuchunjiang1955@sina.com

Key words: meningioma, hemorrhage, magnetic resonance imaging, pathology, mortality intervention are essential to prevent further episodes of bleeding, which may otherwise have fatal consequences for the patients.

\section{Introduction}

Meningiomas are the most commonly occurring primary intracranial tumors, accounting for 20.0-33.8\% of all intracranial tumors $(1,2)$. According to the WHO histological classification, tumors may be classified as benign (grade 1), atypical (grade 2) or malignant (grade 3) $(3,4)$. Atypical and malignant meningiomas account for $\sim 5.0 \%$ of all meningiomas (2). Although meningiomas are mostly benign, the probability of an associated hemorrhage is $1.3-2.4 \%$ (5-7). Hemorrhage can occur within the tumors, surrounding the tumors, and within the brain parenchyma, subarachnoid space and subdural space (8-10). During such events, substantial bleeding may lead to severe clinical consequences. Cheng and Lin (11) reported that the mortality of hemorrhagic meningioma was as high as $38.5 \%$ in the computed tomography (CT) era and $77.8 \%$ in the pre-CT era. Bosnjak et al (12) reported that the overall mortality and morbidity rates of hemorrhagic meningioma were 21.1 and $32.6 \%$ in 2001 , respectively.

The exact causes of hemorrhage in meningioma are currently unknown. The most common hypotheses are infarction of the tumor with secondary bleeding, increased density of blood vessels inside the tumor, direct tumor invasion into one of the cerebral arteries, mechanical stretching and distortion of the cortical bridging veins, and histamine-related vasodilatation or venous hypertension due to occlusion of the venous sinus $(6,13)$.

In the present study, a case of sphenoidal ridge meningioma with repeated bleeding episodes, manifested as intratumoral parenchymal hemorrhages, is described. The progressive process eventually resulted in the formation of a cerebral hernia, which played an important role in the causal analysis of the hemorrhage. In addition, a literature review on data between 2006 and 2019 was performed to provide supplemental information on the causes of meningioma hemorrhage. 


\section{Case report}

Medical history. A 35-year-old female patient was admitted to the Sanbo Brain Hospital of the Capital Medical University (Beijing, China) on November 19, 2018, with a complaint of an intermittent week-long headache, aggravated nausea and vomiting. The patient had experienced episodes of intermittent headache 1 week prior to the aforementioned symptoms and had been admitted to a local hospital. CT examination of the head showed hemorrhagic stroke, following which, mannitol dehydration treatment $(20 \%$, once every $12 \mathrm{~h}$; $125 \mathrm{ml}$ each time) was applied. After 4 days, the patient started showing aggravated symptoms, accompanied by nausea and vomiting. A repeat head CT demonstrated aggravated hemorrhage. The patient was then transferred to Sanbo Brain Hospital. Upon admission, the nervous system examination results were as follows: A Glasgow Coma Scale score (14) of 13 , lethargy, motor aphasia, unequal pupil size (left, $5.0 \mathrm{~mm}$; right, $2.0 \mathrm{~mm}$ ), disappearance of the left light reflex and a responsive right light reflex. The muscular strength of the right limb was grade III (Medical Research Council sum score) (15), tension was increased, deep reflex was hyperactive and right pathological reflex was positive. Admission blood tests revealed a normal platelet count, hemoglobin level, international normalized ratio and activated partial thromboplastin time.

The study was approved by the Ethics Committee of Sanbo Brain Hospital. Written informed consent for publication was obtained from the patient.

Imaging examination. On November 12, 2018, at the beginning of the onset of hemorrhage, the CT examination showed bloody high-density shadows in the left temporal region (Fig. 1A), whereas CT angiography did not show any aneurysms or arteriovenous malformations (Fig. 1D). Another CT scan performed 3 days later showed a significant increase in bleeding as compared with the previous scan, and the blood was observed to have entered the brain parenchyma and have formed a secondary temporal lobe gyrus hernia (Fig. 1B and C). A magnetic resonance imaging (MRI) scan of the head revealed that the left temporal space-occupying lesions showed isointense and hyperintense signals on T1-weighted imaging (Fig. 1E), hyperintense and hypointense signals on T2-weighted imaging and a visible liquid level (Fig. 1F) on November 19, 2018. Susceptibility-weighted imaging (SWI) showed that the hemorrhage site was located in the brain parenchyma and within and around the tumor (Fig. 1G). Enhanced scanning showed that the lesions were uniformly enhanced, whereas the surrounding hematoma was ring-enhanced and displayed the meningeal tail sign (Fig. 1H). Post-surgical CT and MRI examinations showed that the lesions had been completely removed (Fig. 1I and J).

Intraoperative and postoperative conditions. Emergency resection was performed on admission. No adherent veins were found around the tumor. The intratumoral hemorrhage that was causing blood flow into the brain parenchyma was evaluated. After the operation, the patient regained consciousness and limb function gradually recovered. After 9 days, the patient was discharged from hospital. At the time of discharge, the patient could walk independently, but still experienced incomplete aphasia. The left pupil was large $(4.5 \mathrm{~mm})$, but sensitive to light reflection. The patient was then transferred to a rehabilitation hospital for recovery treatment. At the 3-month follow-up, the speech and pupil functions had recovered, and a re-examination with MRI showed no recurrence of the tumor (Fig. 1J).

Histology. Gross morphology of the fresh tumor tissue excised from surgery was fixed in 10\% neutral buffered formalin overnight for histological examinations at room temperature (24-26 $\left.{ }^{\circ} \mathrm{C}\right)$. Furthermore, samples were specially dehydrated, paraffin embedded and sectioned at $5-\mu \mathrm{m}$ thickness following the procedures. After that sections were stained with hematoxylin and eosin (H\&E) and/or immunohistochemical stains. The pathology result was analyzed by 2 experienced specialists in the field of neuropathology according to WHO classification of CNS tumors (2016) (4).

Immunohistochemistry. Gross morphology of the fresh tumor tissue excised from surgery was fixed in $10 \%$ neutral buffered formalin overnight for histological examinations at room temperature. Formalin-fixed sections were deparaffinized and stained with hematoxylin and eosin (H\&E) and immunohistochemistry according to the manufacturers' instructions. In brief, $5-\mu \mathrm{m}$ sections were deparaffinized. The sections were then treated with $3 \% \mathrm{H}_{2} \mathrm{O}_{2}$ for $5 \mathrm{~min}$ at room temperature to block endogenous peroxidase activity. Antigen retrieval was performed by steaming the slides in target retrieval solution, citrate $\mathrm{pH} 6$ (Agilent Technologies Inc.) for $15 \mathrm{~min}$ and then cooling for $15 \mathrm{~min}$. Then, the slides were blocked with $5 \%$ fetal bovine serum (Jackson ImmunoResearch Laboratories, Inc.) diluted in wash buffer with $1 \%$ bovine serum albumin (Merk KGaA) at room temperature for $15 \mathrm{~min}$ and incubated with primary antibodies against epithelial membrane antigen (EMA; 1:100; cat. no. E29; DAKO, Agilent Technologies, Inc.), Ki-67 (1:50 dilution; cat. no. MIB-1; Labvision), progesterone receptor (PR; 1:150 dilution; cat. no. ab8327; Abcam); CD34 (1:100; cat. no. ab8158; Abcam); vimentin (1:150; cat. no. ab9254; Abcam) at $4^{\circ} \mathrm{C}$ overnight. After being washed with phosphate-buffered saline 3 times, the sections were stained with anti-mouse/rabbit polymer horse radish peroxidase-labeled secondary antibody (cat. no. PV-6000D; Zhongshan Bio-Tech Co., Ltd) for $30 \mathrm{~min}$ at $37^{\circ} \mathrm{C}$. Then, 3,3'diaminobenzidine (DAB) was applied for color development at room temperature for $5 \mathrm{~min}$ and sections were subsequently counterstained with hematoxylin. Each slide was individually reviewed and scored using Faramount mounting solution (Agilent Technologies Inc.) by 2 experienced neuropathologists using light microscopy (x100-200).

Pathological findings. H\&E-stained section demonstrated diffuse proliferation of spindle shaped tumor cells arranged in bundles and storiform (Fig. 2A), without necrosis, mitosis and invasion of brain tissue. Hemorrhagic and proliferative blood vessels were observed within the tumor (Fig. 2B and C). Therefore, Grade 1 fibrous meningioma was diagnosed, according to the classification criteria of 2016 WHO (4). Ki-67 expression was 5-10\% (Fig. 2D). Immunohistochemical 


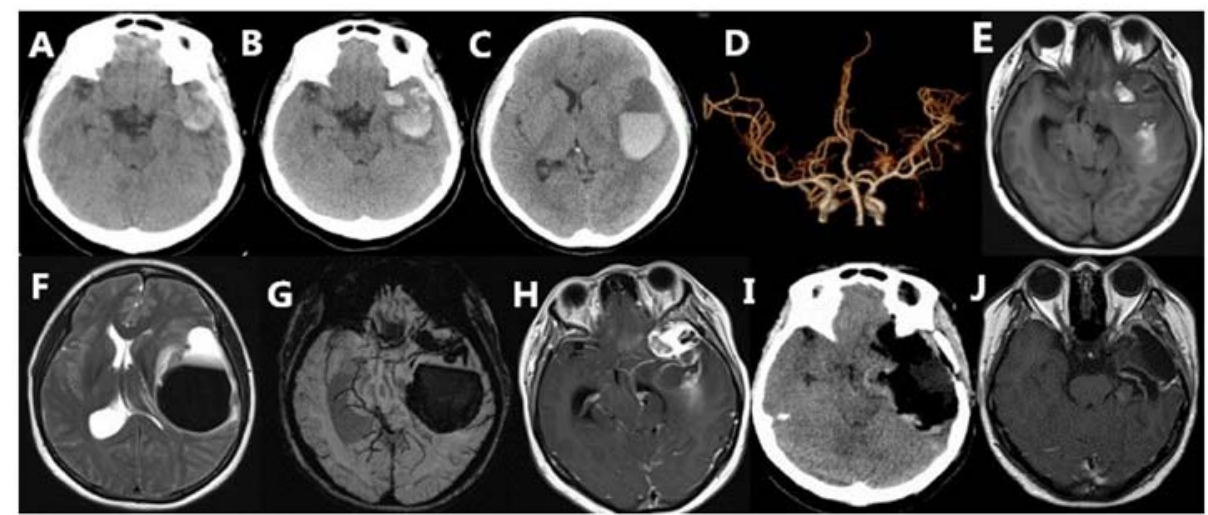

Figure 1. (A) Axial CT scanning showed bloody high-density shadows in the left temporal region, and (B and C) subsequent examination showed a marked increase in hemorrhage. (D) CT angiography revealed no aneurysms or arteriovenous malformations. On axial MRI scans, (E) isointense and hyperintense signals on T1-weighted imaging, (F) hyperintense and hypointense signals on T2-weighted imaging and a visible liquid level were noted. (H) Susceptibility-weighted imaging showed that the hemorrhage site was located in the brain parenchyma and within and around the tumor. (G) Enhanced scanning showed that the lesions were uniformly enhanced, whereas the surrounding hematoma was ring-enhanced and displayed the meningeal tail sign. (I and J) A postoperative review of axial CT and MRI showed that the lesions were completely removed. CT, computed tomography; MRI, magnetic resonance imaging.

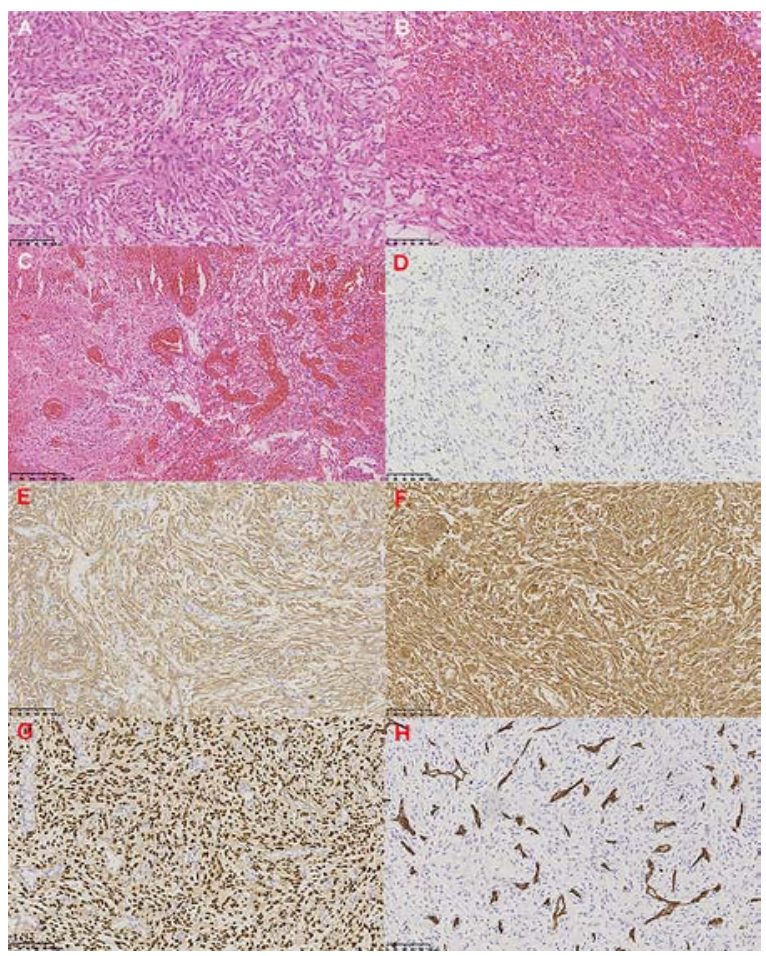

Figure 2. (A) Pathological examination (H\&E staining) confirmed a fibrous meningioma. (B and C) Hemorrhagic and proliferative blood vessels were observed in H\&E-stained tumors. (D) Ki-67 (5-10\%). Immunohistochemical staining showed positive expression of (E) epithelial membrane antigen, (F) vimentin $(\mathrm{F})$ and $(\mathrm{G})$ progesterone. $(\mathrm{H}) \mathrm{CD} 34$ labeling showed increased vascular density. (A), (B), (D-H)=(magnification, $x 200) ; C=($ magnification, $\mathrm{x} 100)$.

staining revealed positive expression of epithelial membrane antigens, vimentin and progesterone (Fig. 2E-G). The CD34 marker demonstrates vascular dysplasia (Fig. 2H).

Literature review on hemorrhagic meningiomas (2006-2019). A total of 40 cases of hemorrhagic meningioma were reviewed (Table I). The mean age of onset was $56.23 \pm 12.91$ years, and the male to female ratio was $1: 1.35$. Cystic changes were observed in $5.88 \%(2 / 34)$ and recurrences were observed in
$11.76 \%(4 / 34)$ of cases. The mortality rate was $9.09 \%(3 / 33)$. Intratumor hemorrhage accounted for $80.00 \%(32 / 40)$ of cases, and $37.50 \%(15 / 40)$ presented with solely intratumoral hemorrhage. Multiple ventricular, peritumoral and subdural hemorrhages also occurred. The proportion of solely subdural hemorrhage cases was $17.50 \%$ (7/40), and only 1 case of solely subarachnoid hemorrhage was reported $(2.5 \%)$. In terms of pathological type, the meningothelial tumor occurred in $34.62 \%(9 / 26)$ of cases (Fig. 3A) and in $45.00 \%(18 / 40)$ of cases convex lesions were mostly observed (Fig. 3B).

\section{Discussion}

Meningiomas are usually benign tumors that arise from arachnoidal cap cells and are detected by the symptoms caused by increased intracranial pressure and seizures. Progressive neurological deficits may also occur, depending on the tumor location and growth (16). Hemorrhagic meningioma is a rare disease with an incidence of 2.0-2.2\% in Asia in the last two decades $(6,7)$. Repeated hemorrhages are rare, with only 2 cases having been reported $(17,18)$. From these cases, plus the present case, meningioma patients with repeated hemorrhages were found to be young, with the average age of onset being $28 \pm 6.24$ years (Table II). One of the previous cases was in a pregnant woman, and a possible cause could be the fluctuation of hormone levels during pregnancy, a hypothesis that has also been supported by other studies $(19,20)$. In the present study, the patient also displayed a high level of progesterone. It was also reported that the internal tumor hemorrhage progressed gradually and eventually broke into the peritumoral area and the brain parenchyma. As tumors have a higher cellular density than the brain parenchyma, it is not surprising that tumoral blood can encroach the brain parenchyma after breaking through the tumor. This may also explain why there are more peritumoral and parenchymal hemorrhages than intratumoral hemorrhages. Another possible cause of hemorrhage was found to be the high proliferation index of tumors. Niiro et al (7) reported that the MIB-1 labeling index of 5 grade I meningioma cases was $5.8 \pm 2.2$. Ki-67 is known to increase with the grade of meningioma and may be 


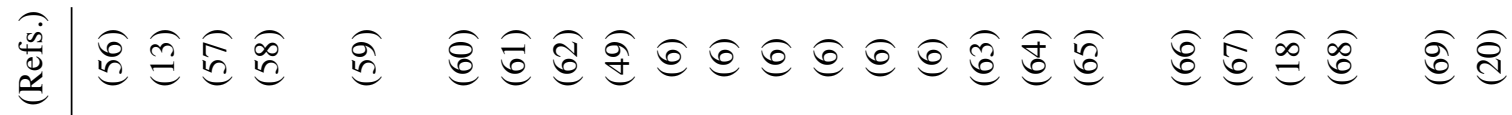
莙 蛋

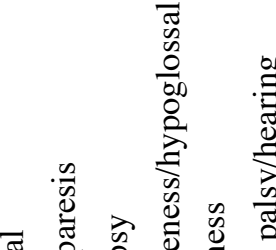

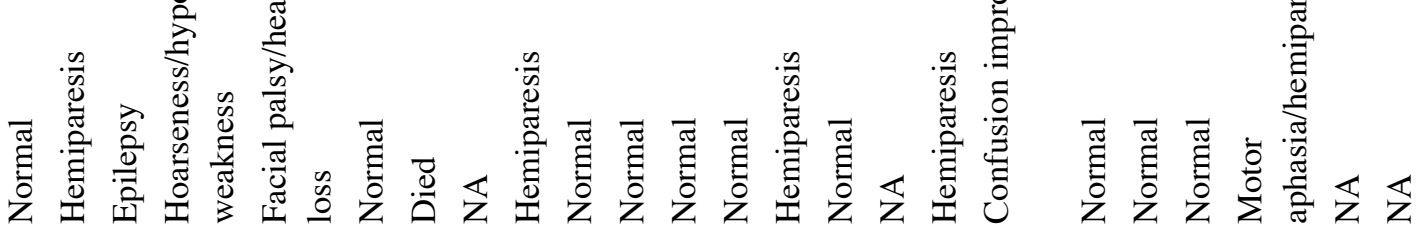

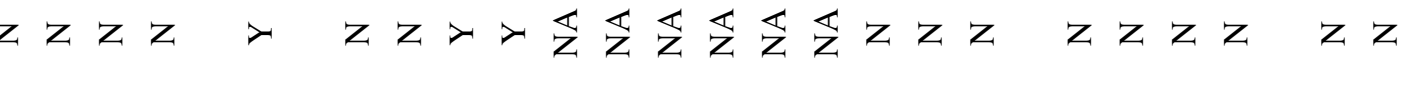

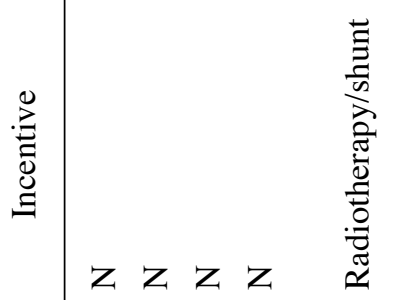

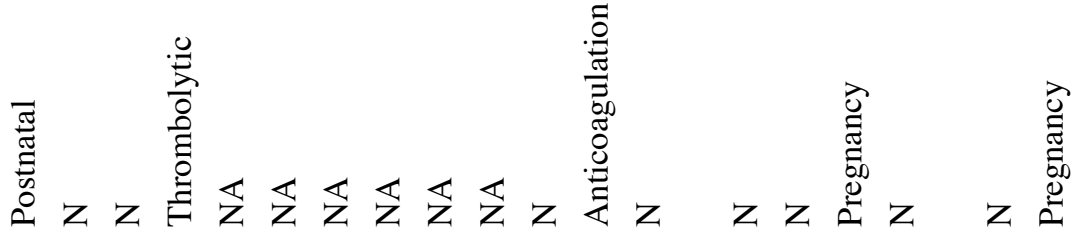

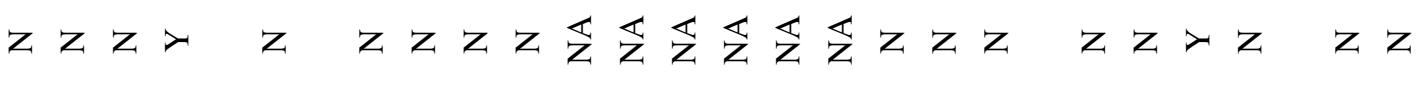

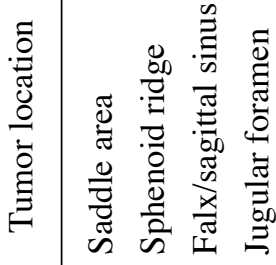

丞䄑声

乙 $\quad$ 乙

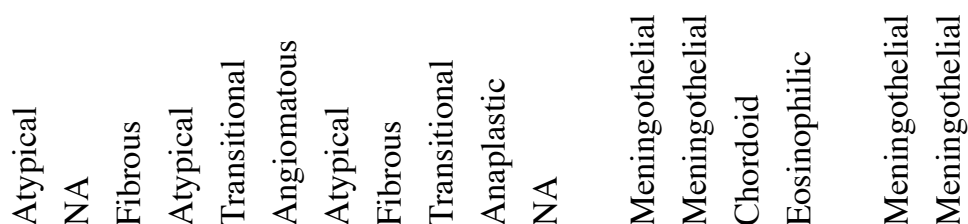
I

$\stackrel{凶}{\omega}$ $\Sigma \Sigma \Sigma \amalg \Sigma$ 票焉志志志

焉

焉焉突焉 $\sum^{\infty} \sum^{\infty} \overline{0}$ 过

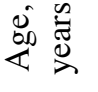

$\underset{m}{\infty} \vec{\infty} i$

ڤิ वे

च $\infty \frac{\infty}{\tilde{\tau}}$

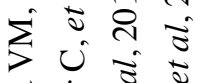
$\Xi$

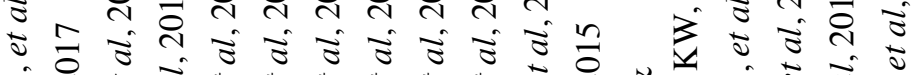

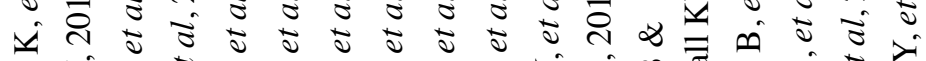

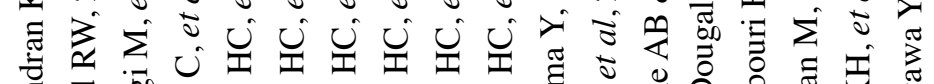

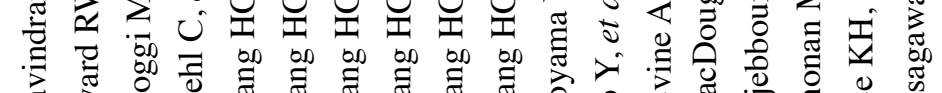
吾 要 


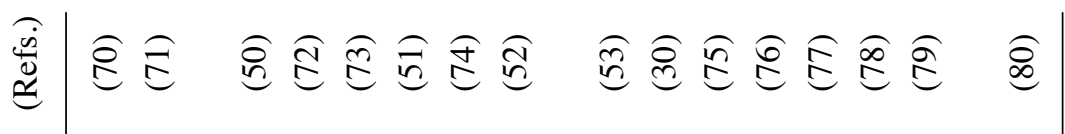

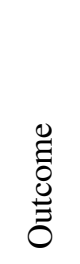

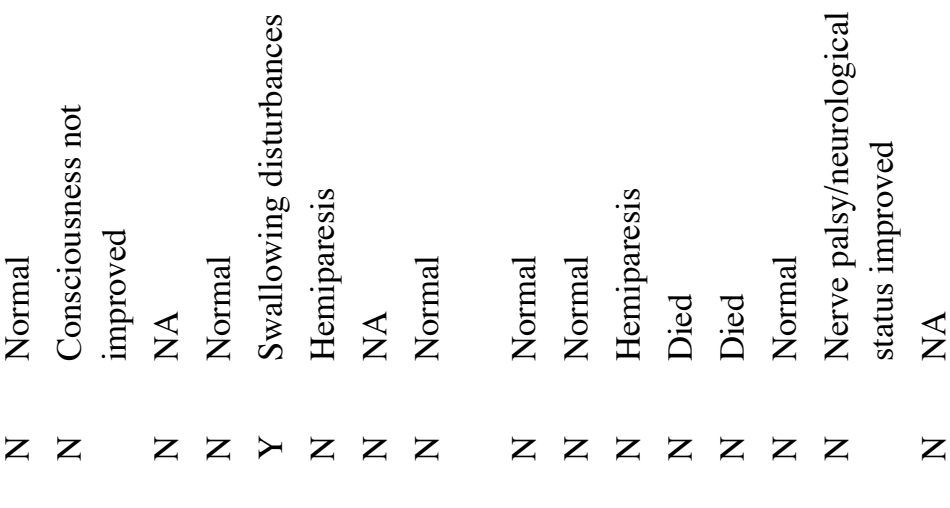

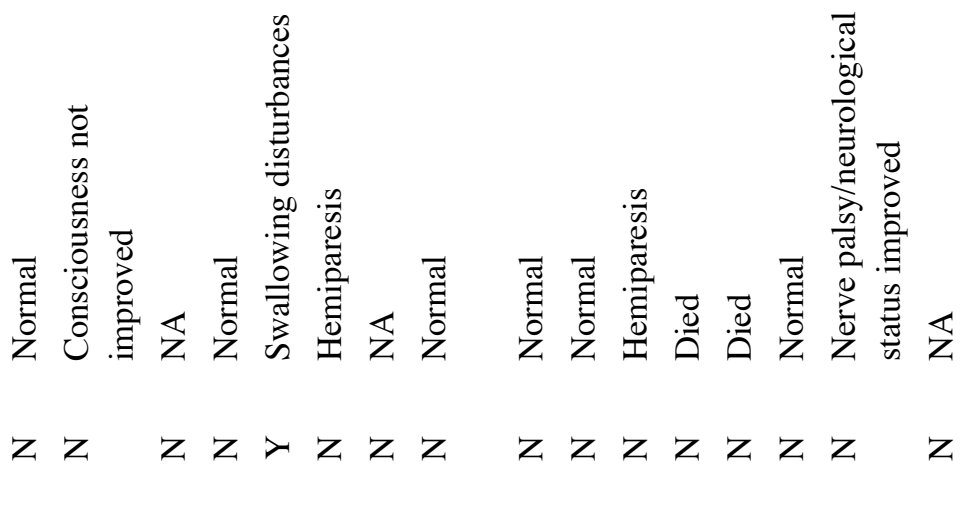

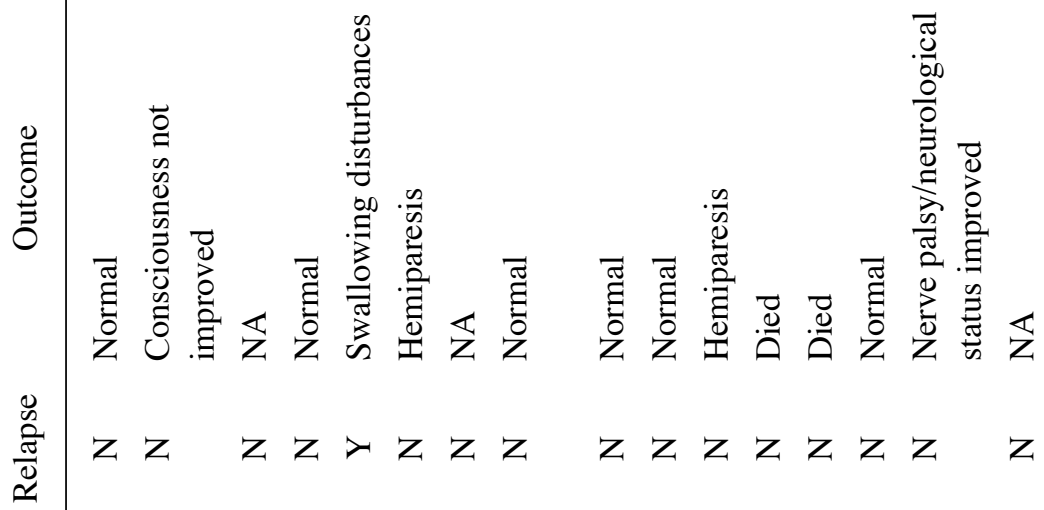

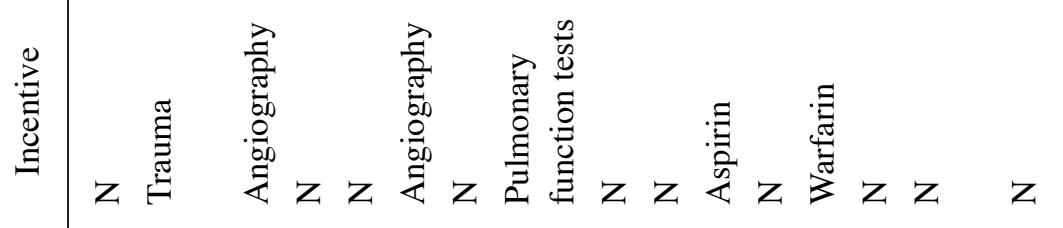
恖

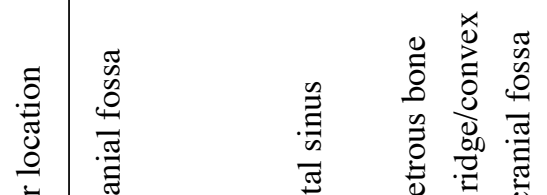

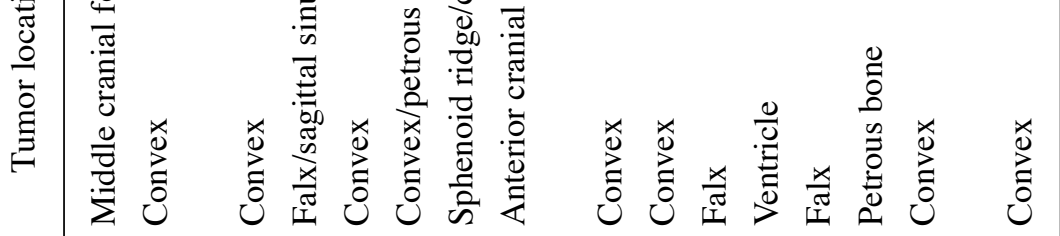

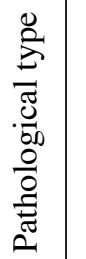

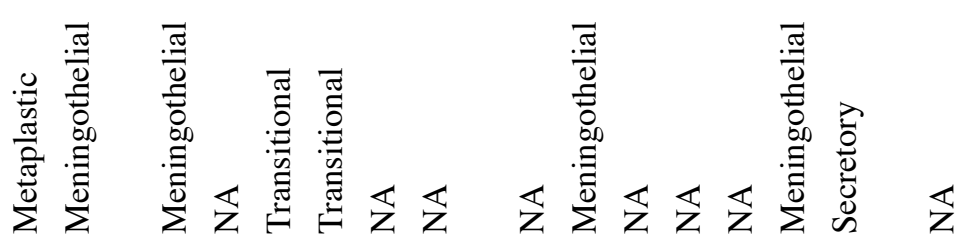
竞

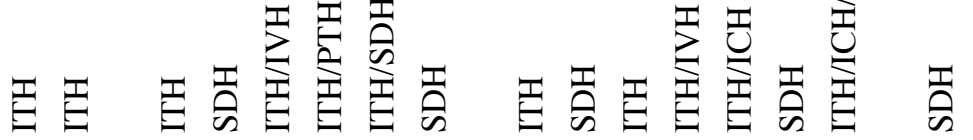

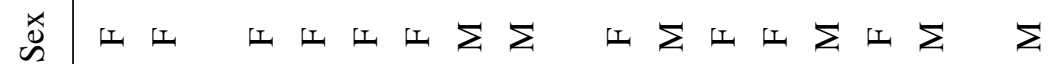

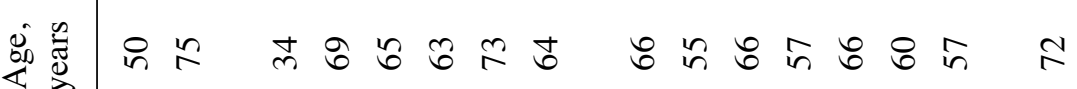

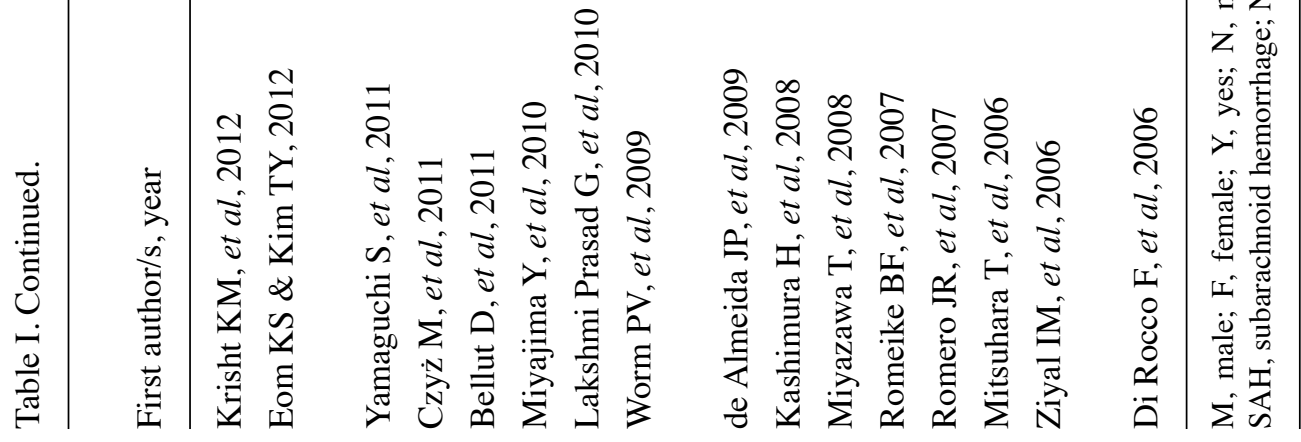


associated with the biological behavior of the invasion $(21,22)$. Sunada et al (23) also reported that a high cell proliferation index may activate certain mechanisms leading to malignant tumor hemorrhage. In addition, abnormal vascular proliferation, as in the case reported in the present study, may also be one of the causes of hemorrhage (Fig. $2 \mathrm{C}$ and $\mathrm{H}$ ).

A review of previous studies revealed that little research has been conducted on the imaging features of meningioma hemorrhage. In most cases, intensive MRI scans have been used; however, T1- and T2-weighted imaging can offer reference support in determining the timing of the hemorrhage. Based on the timeline, intracranial hemorrhage can be classified into hyperacute stage (the first few hours), acute stage (1-3 days), early subacute stage (3-7 days), late subacute stage (4-7 days to 1 month), and chronic stage (1 month to years) $(24,25)$. The bleeding time of meningioma can also be evaluated by MRI signal information. The SWI MRI technique is sensitive to paramagnetic blood products, such as methemoglobin, deoxyhemoglobin, ferritin and hemosiderin. SWI is considered the most sensitive tool available for the detection of cerebral microbleeds (26-28). MRI revealed the subacute hemorrhage in the patient of the present study. The tumor diameter seemed to be unrelated to meningioma hemorrhage, and there are reports of secondary bleeding from small tumors $(29,30)$. In addition, the literature review showed that calcification of hemorrhagic meningiomas is rare (31), which may be due to the high tumor density that makes bleeding difficult.

In 2005, Bosnjak et al (12) conducted a study on 134 cases of hemorrhagic meningioma and showed that factors that may lead to bleeding are a patient age of $<30$ or $>70$ years, convex and intraventricular lesions, and a fibrous tumor type. The mortality rate was reported to be $21.1 \%$. In the present study, 40 cases of hemorrhagic meningioma, reported in the past 14 years, were reviewed and convex meningioma was shown to have a higher probability of hemorrhage. Although numerous pathological types of meningioma can produce hemorrhage, a meningoepithelial tumor is the type most likely to do so. Niiro et al (7) investigated 57 cases of hemorrhagic meningioma, and also showed that meningothelial is the most common pathological type, followed by fibrotic type and hemangiomas. Cystic changes are relatively rare, and were observed in $5.88 \%$ of the hemorrhagic meningioma cases. The incidence of cystic meningiomas was $1.7-10 \%$ (32). It is questionable whether the same disease occurs simultaneously into two separate processes of a rare disease progression and whether there is a correlation between these two processes. However, some reports argue that cystic meningiomas are prone to hemorrhage $(33,34)$. With the development of imaging detection and diagnosis technologies, the mortality rate due to meningiomas has decreased. In the present study, the mortality rate was found to have decreased to $9.09 \%$ in the past 14 years (12).

The precise causes of hemorrhage in meningioma are unknown. The most regularly expounded theories are of tumor infarction with secondary bleeding, direct invasion of the tumor into a cerebral artery, mechanical stretching and distortion of the cortical bridging veins, and histamine-associated vasodilatation or venous hypertension as a result of venous sinus occlusion (13). A recent study have also found that the 
A

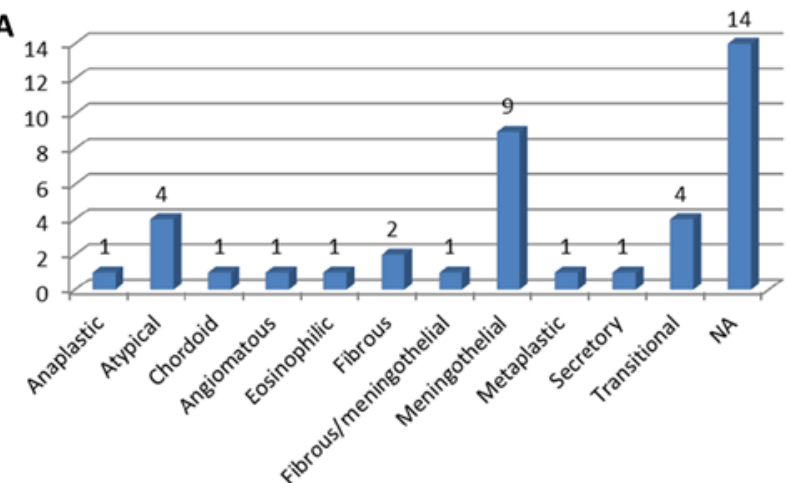

B

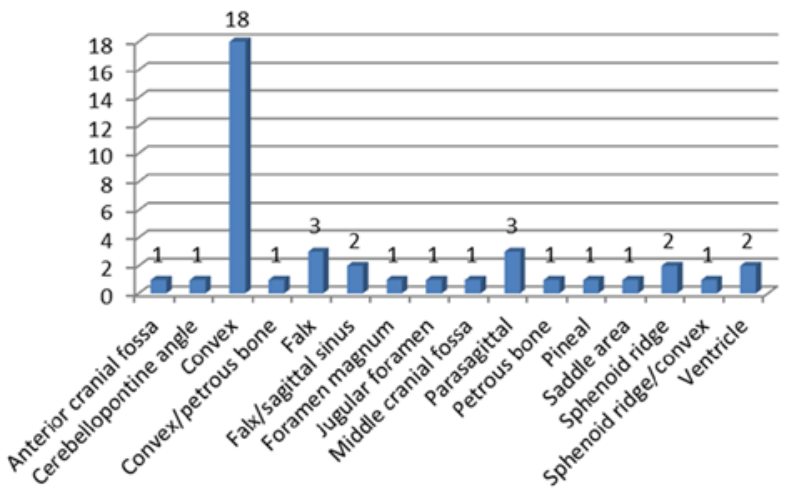

Figure 3. In the literature review, (A) the main pathological type of meningioma hemorrhage was meningothelial tumor, and (B) the location was mainly convex.

average number of undifferentiated vessels in hemorrhagic meningioma patient groups is significantly higher than that in the control groups, thereby confirming their existence as factors involved in the mechanism of hemorrhage (6). In the case report of the present study, the patient showed an onset of intratumor hemorrhage, followed by aggravated bleeding that penetrated into the brain parenchyma. Further analysis of relevant hemorrhage cases (mostly individual cases) in the past 14 years indicated that a majority of hemorrhages occur from inside the tumor, i.e., they are precipitated by an internal factor. Intratumoral hemorrhage is recorded to account for $80.00 \%(32 / 40)$ of cases. In such cases, it is estimated that the hemorrhage may have started inside the tumor and progressed, leading to the blood entering the peritumoral and subdural areas, and even encroaching the brain parenchyma. Due to the high density of the tumor, hemorrhage caused by an external factor, such as a peritumoral blood vessel penetrating into the brain parenchyma, is unlikely. Moreover, in the present study, the literature review focused on evaluating traceable causative factors, including embolization therapy (35-40), pregnancy $(19,20)$, radiotherapy $(41,42)$, air travel $(43)$, surgery (44), trauma (8), long-term cough (45), anticoagulation therapy (46-48), thrombolytic therapy (49), cerebral angiography $(50,51)$ and pulmonary function examination (52). Prevention and intervention targeted at these factors may reduce the probability of hemorrhage.

Hemorrhage of the meninges is often a serious event with a high mortality rate. However, several studies have reported shrinkage in tumor size following a hemorrhagic event (53). Surgical intervention for hemorrhagic meningiomas is the unanimously accepted treatment $(11,54,55)$. In the present case, the patient had a secondary cerebral hernia, which was cured after proactive treatment. Early intervention is necessary to eliminate the risk of secondary bleeding, which can often lead to severe consequences.

In conclusion, repeated hemorrhages in meningiomas are extremely rare and the causes have not been identified yet. Increased Ki-67 and abnormally proliferating blood vessels may be potential causes of hemorrhage. Early diagnosis and rapid surgical intervention are essential to prevent further episodes of bleeding, which may have fatal consequences for the patient. A limitation of the present study is the insufficient number of studies reviewed; however, the rare case reported will add to the scant literature on such cases and will offer a basis for future research in this field.

\section{Acknowledgements}

Not applicable.

\section{Funding}

The study was supported by the Beijing Municipal Science and Technology Commission of China (grant no. Z181100001718199).

\section{Availability of data and materials}

The datasets used or analyzed during the present study are available from the corresponding author on reasonable request.

\section{Authors' contributions}

CJY, CKY and SH designed the study; YKY and NL collected data and revised the manuscript for important intellectual content; XLQ performed the histological examination of the samples; ZCY analyzed the data and SH wrote the article. All authors have read and approved the final manuscript.

\section{Ethics approval and consent to participate}

The study was approved by the Ethics Committee of Sanbo Brain Hospital (Beijing, China).

\section{Patient consent for publication}

Written informed consent was obtained from the patient.

\section{Competing interests}

The authors declare that they have no competing interests.

\section{References}

1. Claus EB, Bondy ML, Schildkraut JM, Wiemels JL, Wrensch M and Black PM: Epidemiology of intracranial meningioma. Neurosurgery 57: 1088-1095, 2005. 
2. Wiemels J, Wrensch M and Claus EB: Epidemiology and etiology of meningioma. J Neurooncol 99: 307-314, 2010.

3. Louis DN, Ohgaki H, Wiestler OD, Cavenee WK, Burger PC, Jouvet A, Scheithauer BW and Kleihues P: The 2007 WHO classification of tumours of the central nervous system. Acta Neuropathol 114: 97-109, 2007.

4. Louis DN, Perry A, Reifenberger G, von Deimling A, Figarella-Branger D, Cavenee WK, Ohgaki H, Wiestler OD, Kleihues P and Ellison DW: The 2016 World Health Organization classification of tumors of the central nervous system: A summary. Acta Neuropathol 131: 803-820, 2016.

5. Bromowicz J: Spontaneous hemorrhage in brain tumors. Neurol Neurochir Pol 17: 471-475, 1983 (In Polish).

6. Wang HC, Wang BD, Chen MS, Li SW, Chen H, Xu W and Zhang JM: An underlying pathological mechanism of meningiomas with intratumoral hemorrhage: Undifferentiated microvessels. World Neurosurg 94: 319-327, 2016.

7. Niiro M, Ishimaru K, Hirano H, Yunoue S and Kuratsu J: Clinico-pathological study of meningiomas with haemorrhagic onset. Acta Neurochir (Wien) 145: 767-72, 2003.

8. Bruno MC, Santangelo M, Panagiotopoulos K, Piscopo GA, Narciso N, Del Basso De Caro MI, Briganti F and Cerillo A: Bilateral chronic subdural hematoma associated with meningioma. Case report and review of the literature. J Neurosurg Sci 47: 215-27, 2003.

9. Martínez-Lage JF, Poza M, Martínez M, Esteban JA, Antúnez MC and Sola J: Meningiomas with haemorrhagic onset. Acta Neurochir (Wien) 110: 129-32, 1991.

10. Jones NR and Blumbergs PC: Intracranial haemorrhage from meningiomas: A report of five cases. Br J Neurosurg 3: 691-698, 1989.

11. Cheng MH and Lin JW: Intracranial meningioma with intratumoral hemorrhage. J Formos Med Assoc 96: 116-120, 1997.

12. Bosnjak R, Derham C, Popović M and Ravnik J: Spontaneous intracranial meningioma bleeding: Clinicopathological features and outcome. J Neurosurg 103: 473-484, 2005.

13. Mandour C, Laaguili J, Gazzaz M and Mostarchid BE: Spontaneous intraventricular hemorrhage caused by sphenoid meningioma. J Neurol Surg A Cent Eur Neurosurg 79: 434-435, 2018.

14. Teasdale G and Jennett B: Assessment of coma and impaired consciousness. A practical scale. Lancet 2: 81-84, 1974.

15. Kleyweg RP, van der Meché FG and Schmitz PI: Interobserver agreement in the assessment of muscle strength and functional abilities in Guillain-Barré syndrome. Muscle Nerve 14: 1103-1109, 1991

16. Kouyialis AT, Stranjalis G, Analyti R, Boviatsis EJ, Korfias S and Sakas DE: Peritumoural haematoma and meningioma: A common tumour with an uncommon presentation. J Clin Neurosci 11: 906-909, 2004.

17. Scotti G, Filizzolo F, Scialfa G, Tampieri D and Versari P: Repeated subarachnoid hemorrhages from a cervical meningioma. Case report. J Neurosurg 66: 779-781, 1987.

18. Lee KH, Lall RR, Chandler JP, Bigio EH and Mao Q: Pineal chordoid meningioma complicated by repetitive hemorrhage during pregnancy: Case report and literature review. Neuropathology 33: 192-198, 2013.

19. Chow MS, Mercier PA, Omahen DA, Wood SL and Johnson JA Recurrent exophytic meningioma in pregnancy. Obstet Gynecol 121 (Suppl 1): S475-S478, 2013.

20. Kumar S, Gupta V and Khandelwal N: Hemorrhage in meningioma: An unwanted outcome of pregnancy. Neurol India 61: 329-330, 2013.

21. Bohra H, Rathi KR, Dudani S, Bohra A, Vishwakarma S and Sahai K: The study of MIB-1 LI and CD 34 as a marker of proliferative activity and angiogenesis in different grades of meningioma. J Clin Diagn Res 10: EC14-EC17, 2016.

22. Olar A, Wani KM, Sulman EP, Mansouri A, Zadeh G, Wilson CD, DeMonte F, Fuller GN and Aldape KD: Mitotic index is an independent predictor of recurrence-free survival in meningioma. Brain Pathol 25: 266-275, 2015.

23. Sunada I, Nakabayashi H, Matsusaka Y and Yamamoto S: Meningioma associated with acute subdural hematoma-case report. Radiat Med 16: 483-486, 1998.

24. Smith EE, Rosand J and Greenberg SM: Hemorrhagic stroke. Neuroimaging Clin N Am 15: 259-272, ix, 2005.

25. Parizel PM, Makkat S, Van Miert E, Van Goethem JW, van den Hauwe L and De Schepper AM: Intracranial hemorrhage: Principles of CT and MRI interpretation. Eur Radiol 11: $1770-1783,2001$
26. Ma X, Bai Y, Lin Y, Hong X, Liu T, Ma L, Haacke EM, Zhou J, Wang $\mathrm{J}$ and Wang $\mathrm{M}$ : Amide proton transfer magnetic resonance imaging in detecting intracranial hemorrhage at different stages: A comparative study with susceptibility weighted imaging. Sci Rep 7: 45696, 2017.

27. Haacke EM, Mittal S, Wu Z, Neelavalli J and Cheng YC: Susceptibility-weighted imaging: Technical aspects and clinical applications, part 1. AJNR Am J Neuroradiol 30: 19-30, 2009.

28. MittalS,WuZ,NeelavalliJandHaackeEM: Susceptibility-weighted imaging: Technical aspects and clinical applications, part 2. AJNR Am J Neuroradiol 30: 232-252, 2009.

29. Sato K, Sugawara T, Fujiwara S, Mizoi K and Yoshimoto T: A case of small meningioma with acute subdural hematoma. No Shinkei Geka 17: 687-690, 1989 (In Japanese).

30. Kashimura H, Arai H, Ogasawara K, Beppu T, Kurose A and Ogawa A: Lipomatous meningioma with concomitant acute subdural hematoma-case report-. Neurol Med Chir (Tokyo) 48: 466-469, 2008

31. Levy A and Mansuy L: Two cases of cerebral tumors with atypical calcification; chronic subdural meningioma and hematoma. J Radiol Electrol Arch Electr Medicale 32: 821-822, 1951 (In Undetermined Language).

32. Wang P, Han S, Liu N, Yu C, Qi X, Zhu M, Zhang X, Wang LI and Yan C: Peritumoral cystic meningioma: A report of two cases and review of the literature. Exp Ther Med 11: 904-908, 2016.

33. Wakamoto H, Miyazaki H, Hayashi T, Shimamoto $\mathrm{Y}$ and Ishiyama $\mathrm{N}$ : Intratumoral hemorrhage associated with cystic meningioma under observation: A case report. No Shinkei Geka 26: 247-252, 1998 (In Japanese).

34. Kuzeyli K, Cakir E, Usul H, Karaarslan G, Yazar U, Baykal S, Reis A and Cobanoglu U: Intratumoral haemorrhage: A clinical study. J Clin Neurosci 11: 490-192, 2004.

35. Ishihara H, Ishihara $S$, Niimi J, Neki H, Kakehi Y, Uemiya N, Kohyama S, Yamane F, Kato H, Suzuki T, et al: The safety and efficacy of preoperative embolization of meningioma with N-butyl cyanoacrylate. Interv Neuroradiol 21: 624-630, 2015.

36. Yu SC, Boet R, Wong GK, Lam WW and Poon WS Postembolization hemorrhage of a large and necrotic meningioma. AJNR Am J Neuroradiol 25: 506-508, 2004

37. Kallmes DF, Evans AJ, Kaptain GJ, Mathis JM, Jensen ME, Jane JA and Dion JE: Hemorrhagic complications in embolization of a meningioma: Case report and review of the literature Neuroradiology 39: 877-880, 1997.

38. Motozaki T, Otuka S, Sato S, Nakao S, Ban S, Fukumitsu T and Yamamoto T: Preoperative embolization with gelfoam powder for intracranial meningioma causing unusual peritumoral hemorrhage-with reference to the mechanism of hemorrhage. No Shinkei Geka 15: 95-101, 1987 (In Japanese).

39. Suyama T, Tamaki N, Fujiwara K, Hamano S, Kimura M and Matsumoto S: Peritumoral and intratumoral hemorrhage after gelatin sponge embolization of malignant meningioma: Case report. Neurosurgery 21: 944-946, 1987

40. Watanabe K, Matsumura K, Matsuda $\mathbf{M}$ and Handa $\mathrm{J}$. Meningioma with intratumoral and subdural hemorrhage as an immediate complication of therapeutic embolization Case report. Neurol Med Chir (Tokyo) 26: 904-907, 1986 (In Japanese).

41. Kim CH, Kim DG, Paek SH, Chung HT, Choi YL and Chi JG: Delayed bleeding after gamma knife surgery for meningioma. Acta Neurochir (Wien) 146: 741-742, 2004

42. Kwon Y, Ahn JS, Jeon SR, Kim JH, Kim CJ, Lee JK, Kwun BD, Lee DH and Kim SY: Intratumoral bleeding in meningioma after gamma knife radiosurgery. J Neurosurg 97 (5 Suppl): S657-S662, 2002.

43. Goldberg CR and Hirschfeld A: Hemorrhage within brain tumors in association with long air travel. Acta Neurochir (Wien) 144: 289-293, 2002

44. Maeda K, Gotoh H, Chikui E and Furusawa T: Intratumoral hemorrhage from a posterior fossa tumor after cardiac valve surgery-case report. Neurol Med Chir (Tokyo) 41: 548-550, 2001.

45. Bloomgarden GM, Byrne TN, Spencer DD and Heafner MD: Meningioma associated with aneurysm and subarachnoid hemorrhage: Case report and review of the literature. Neurosurgery 20: 24-26, 1987.

46. Spektor S, Ashkenazi E and Israel Z: Intracranial haemorrhage from a meningioma in a patient receiving aspirin prophylaxis: A case report. Acta Neurochir (Wien) 134: 51-53, 1995. 
47. Toledo E, Shalit MN and Segal R: Spinal subdural hematoma associated with anticoagulant therapy in a patient with spinal meningioma. Neurosurgery 8: 600-603, 1981.

48. Everett BA, Kusske JA and Pribram HW: Anticoagulants and intracerebral hemorrhage from an unsuspected meningioma. Surg Neurol 11: 233-235, 1979.

49. Diehl C, Haux D, Sahm F, Unterberg AW and Beynon C: Intracranial tumour haemorrhage following intravenous thrombolysis. J Clin Neurosci 26: 145-146, 2016.

50. Yamaguchi S, Suzuki SO, Matsuo Y, Uesaka T, Matsukado K and Iwaki T: Exacerbation of radiation induced meningioma due to hemorrhage after cerebral angiography: A case report. No Shinkei Geka 39: 45-50, 2011 (In Japanese).

51. Miyajima Y, Oka H, Utsuki S, Shimizu S, Suzuki S and Fujii K: Intracranial hemorrhage associated with convexity meningioma after cerebral angiography-case report. Neurol Med Chir (Tokyo) 50: 67-70, 2010.

52. Worm PV, Ferreira MP, Ferreira NP and Cechetti F: Subdural haematoma in a patient with meningioma. Arq Neuropsiquiatr 67 308-310, 2009.

53. de Almeida JP, Petteys RJ, Sciubba DM, Gallia GL and Brem H Regression of intracranial meningioma following intratumoral hemorrhage. J Clin Neurosci 16: 1246-1249, 2009.

54. Okuno S, Touho H, Ohnishi $\mathrm{H}$ and Karasawa J: Falx meningioma presenting as acute subdural hematoma: Case report. Surg Neurol 52: 180-184, 1999.

55. Kohli CM and Crouch RL: Meningioma with intracerebral hematoma. Neurosurgery 15: 237-240, 1984.

56. Ravindra VM, Gozal YM, Palmer C and Couldwell WT: Hemorrhagic atypical planum sphenoidale meningioma with intermittent vision loss-rare presentation of apoplexy. World Neurosurg 121: 71-76, 2019.

57. Hu S, Zhang Y, Sun Y, Yu Y, Wang J, Dai H, Sun F and Hu C: Lung metastases from intracranial bleeding meningioma: A case report. Medicine (Baltimore) 97: e0457, 2018.

58. Basil G, Urakov T, Knudsen MG and Morcos J: Jugular tubercle meningioma with hemorrhagic conversion mimicking a ruptured thrombosed giant vertebrobasilar aneurysm. World Neurosurg 119: 108-112, 2018

59. Entezami P, Riccio A and Kenning TJ: Intratumoral hemorrhage within petrous meningioma. World Neurosurg 117: 246-248, 2018.

60. Ravindran K, Gaillard F and Lasocki A: Spontaneous subdural haemorrhage due to meningioma in the post-partum setting. J Clin Neurosci 39: 77-79, 2017.

61. Byard RW: Parasagittal meningioma: A not so benign entity. Med Sci Law 57: 175-178, 2017.

62. Broggi M, Restelli F and Acerbi F: Role of tumor vessels' features in determining risk of bleeding in Meningiomas: Which came first, the Chicken or the Egg. World Neurosurg 95: 590-593, 2016

63. Aoyama Y, Ohta S, Sakaki S and Fujita T: Foramen magunum meningioma presented as subarachnoid haemorrhage. No Shinkei Geka 43: 445-450, 2015 (In Japanese).

64. Ito Y, Nakajima M, Watari M, Sakamoto T, Hashimoto Y, Tajiri S, Takada A and Ando Y: Intratumoral hemorrhage in a patient with malignant meningioma under anticoagulant therapy. J Stroke Cerebrovasc Dis 24: e91-e92, 2015.

65. Levine AB and MacDougall KW: Subdural hematoma: A rare presentation of a convexity meningioma. Can J Neurol Sci 41: 506-507, 2014
66. Eljebbouri B, Mandour C and ElMostarchid B: Acute headache originating from a bleeding convexity meningioma. Headache 54 : 1222-1223, 2014

67. Chonan M, Niizuma K, Koyama S, Kon H, Sannohe S, Kurotaki H, Midorikawa H, Sasaki T and Nishijima M: An operated case of a meningioma causing acute subdural hematoma. No Shinkei Geka 41: 235-239, 2013 (In Japanese).

68. Sasagawa Y, Tachibana O, Iida T and Iizuka H: Oncocytic meningioma presenting with intratumoral hemorrhage. J Clin Neurosci 20: 1622-1624, 2013.

69. Rocha AJ, Saade N and Silva AZ: Meningioma associated with non-traumatic subdural hematoma: An outstanding appearance of this common intracranial tumor. Arq Neuropsiquiatr 71: 417, 2013.

70. Krisht KM, Altay T and Couldwell WT: Myxoid meningioma: A rare metaplastic meningioma variant in a patient presenting with intratumoral hemorrhage. J Neurosurg 116: 861-865, 2012.

71. Eom KS and Kim TY: Isolated hemorrhagic contusion of an incidental meningioma. Ulus Travma Acil Cerrahi Derg 18: 277-279, 2012.

72. Czyż M, Jarmundowicz W, Szarek D, Tabakow P and Markowska-Wojciechowska A: Bilateral chronic subdural haematomas in a patient with meningioma of the superior sagittal sinus-case report and pathophysiological study. Neurol Neurochir Pol 45: 500-504, 2011

73. Bellut D, Nern C, Burkhardt JK, Könü D, Bertalanffy H and Krayenbühl N: Acute recurrent haemorrhage of an intracranial meningioma. J Clin Neurosci 18: 992-993, 2011.

74. Lakshmi Prasad G, Ramdurg SR, Suri A and Mahapatra AK: A rare association of meningioma with intratumoral bleed and acute subdural hematoma. Neurol India 58: 977-978, 2010.

75. Miyazawa T, Uozumi Y, Toyooka T and Shima K: Hemorrhage from a falx meningioma after internal use of low-dose aspirin. J Stroke Cerebrovasc Dis 17: 325-327, 2008.

76. Romeike BF, Joellenbeck B, Skalej M, Scherlach C, Kirches E and Mawrin C: Intraventricular meningioma with fatal haemorrhage: Clinical and autopsy findings. Clin Neurol Neurosurg 109: 884-887, 2007.

77. Romero JR, Sakai O, Rice MB and Babikian VL: Intracranial hemorrhage sparing meningioma in an anticoagulated patient. J Neuroimaging 17: 246-250, 2007.

78. Mitsuhara T, Ikawa F, Ohbayashi N, Imada Y, Abiko M and Inagawa T: A case of petrotentorial meningioma presented as an acute subdural hemorrhage. No Shinkei Geka 34: 827-832, 2006 (In Japanese).

79. Ziyal IM, Bilginer B, Celik O, Ozcan OE and Ozgen T: Tentorial meningioma on follow-up presenting with sudden deterioration due to intra- and peritumoral hemorrhage. Acta Neurochir (Wien) 148: 1315-1316, 2006.

80. Di Rocco F, Mannino S, Puca A, Lauriola L and Pompucci A: Intracranial meningiomas associated with non-traumatic chronic subdural hematoma. Acta Neurochir (Wien) 148: 1097-1102, 2006.

This work is licensed under a Creative Commons

Attribution-NonCommercial-NoDerivatives 4.0 International (CC BY-NC-ND 4.0) License. 